\title{
Further Observations in Cerebro-Costo-Mandibular Syndrome: Exploration with CT Imaging
}

\author{
Venkatraman Bhat $^{1}$ (1) Vinay Belval ${ }^{1}$ Santhosh Kumar ${ }^{1}$ Onkar Auti ${ }^{1}$ \\ ${ }^{1}$ Department of Radiology, Narayana Health, Bengaluru, India \\ Address for correspondence Venkatraman Bhat, MD, FRCR, 309, \\ J Child Sci 2020;10:e21-e24. \\ Greenwoods Apt, Royal Gardenia, Bommasandra, Bengaluru, 560099 \\ India (e-mail: bvenkatraman@gmail.com).
}

\begin{abstract}
Keywords

- cerebro-costomandibular syndrome

- posterior rib ossification gaps

- CT imaging

Cerebro-costo-mandibular syndrome (CCMS) is a rare syndrome characterized by posterior rib ossification gaps and orofacial anomalies. Posterior rib ossification gaps are the hallmark of the disease and mimic multiple old rib fractures. The condition is caused by heterogeneous mutation of Small Nuclear Ribonucleoprotein Polypeptides B and B1 (SNRPB) gene on the chromosome 20p13. There is scanty literature regarding the extent of bony changes and the associated pulmonary-airway abnormalities on computed tomography (CT) imaging. In this case report, we presented a detailed CT imaging features of a case of CCMS in a male infant. This report highlighted on the nature of osseous defects, structural changes in the chest wall, airways, lungs, and a wide range of spinal anomalies. Some of our observations regarding the changes in the airway and chest wall are likely to have bearing on evaluating the prognosis and the need for respiratory support.
\end{abstract}

\section{Introduction}

Cerebro-costo-mandibular syndrome (CCMS) is a rare syndrome characterized by micrognathia, orofacial anomalies, and posterior rib ossification gaps, with or without mental retardation. Male and female are equally affected. ${ }^{1}$ This condition was first described in 1966 by Smith et al. ${ }^{2}$ Although most cases are spontaneous, both autosomal recessive and autosomal dominant patterns of inheritance have been observed in familial cases. Defects in the SNRPB gene on the chromosome20p13 are responsible for these developmental anomalies (OMIM: 117650). ${ }^{1,3}$ Differential diagnoses of the condition include trisomy 13 and 18 and Pierre Robin sequence. Most of the orofacial anomalies and airway changes appear to represent Pierre Robin sequence and lead to respiratory difficulty and early mortality. ${ }^{4}$ Severity and presentation of the cases vary according to the extent of bone defects, airway integrity, and overall efficiency of ventilatory apparatus. Classically, upper airways are most affected. In most of the previously reported cases, descriptions of chest wall and airway changes were based on plain radiography. Our study, based on threedimensional (3D) volumetric computed tomography (CT) data, provided additional insight and better clarity to airway and

received

November 23, 2019 accepted after revision March 9, 2020 chest wall changes. We also reported extensive anomalies of spine observed in our patient.

\section{Case Report}

A male patient of 1 year and 7 months was referred to the imaging services to exclude spinal anomalies. Patient had clinical features of facial dysmorphism, central hypotonia, developmental motor delay, and microcephaly. The patient was not in respiratory distress. On examination, there was marked mandibular hypoplasia. No defects were noted in soft and hard palate. Clinical examination of chest and abdomen was normal. There was generalized hypotonia. No scoliosis or limb defects were seen. Patient was born at a private hospital. On assessment at birth, the patient had facial dysmorphism, microcephaly, and generalized hypotonia. He did not have respiratory difficulty, and was kept on room air. He underwent plain chest radiography that showed multiple posterior rib defects and suspicion of spina bifida in cervical region. He had also undergone a routine neurosonogram, which was reported as normal. No evidence of intracranial hemorrhage, hydrocephalus, or gross structural anomaly was reported.
Copyright @ 2020 Georg Thieme Verlag License terms
KG Stuttgart · New York 


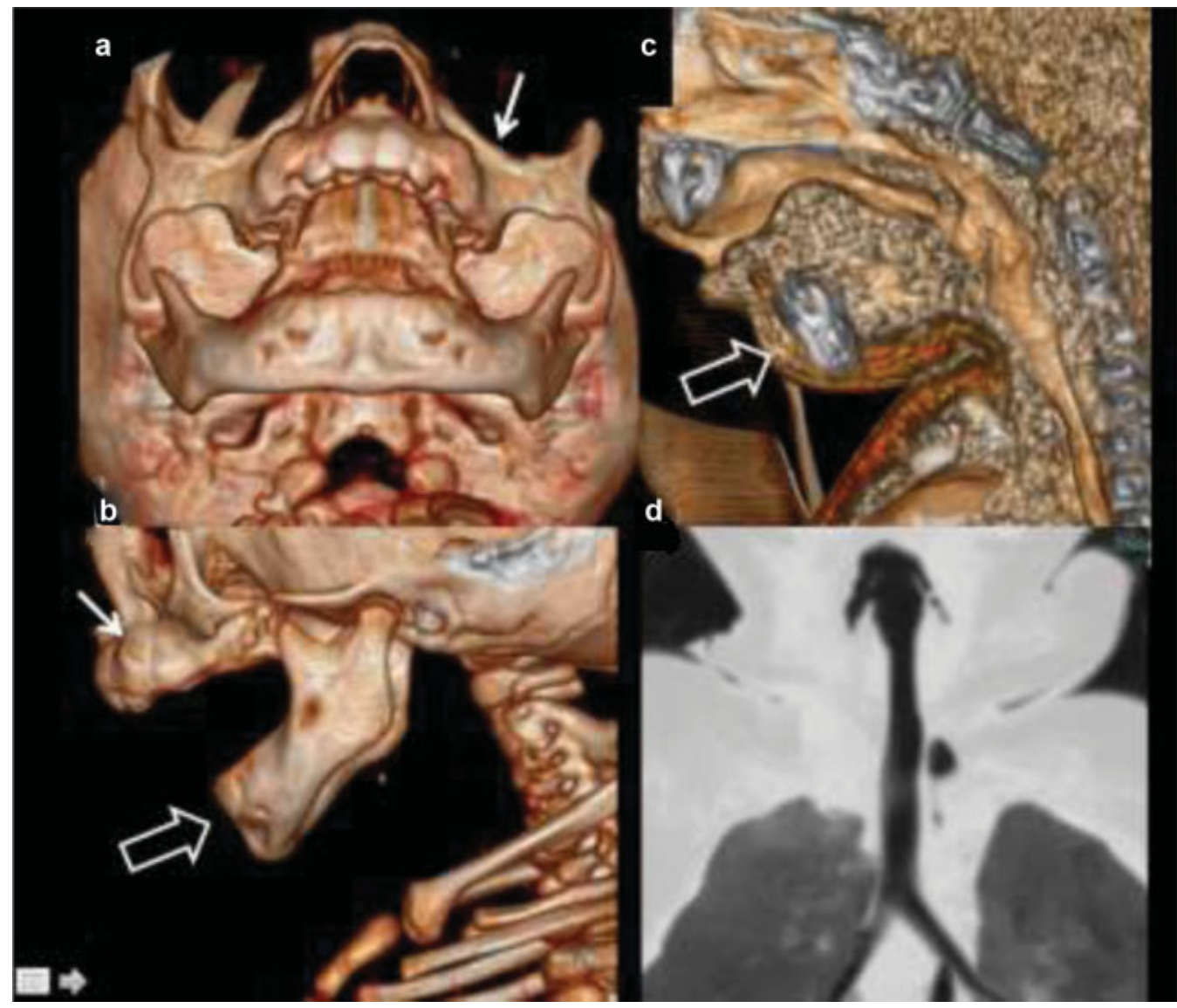

Fig. 1 Three-dimensional rendered images of facial bony structures $(A-C)$ in frontal and lateral perspective show hypoplastic maxillae (arrow) and mandible (open arrow). Airway capacity of upper airways is satisfactory (C and D).

At our institution, the child underwent noncontrast CT examination of thorax and spine to look for airways and any additional structural anomalies in the spine (helical, $100 \mathrm{Kv}$, $100 \mathrm{mAs}$, CTDI vol $3.9 \mathrm{mGy}$, BODY32cm). Multiple radiological findings were identified. Evaluation of face revealed severe micrognathia (-Fig. 1A-C). Hard, soft palate and both external auditory canals were normal. Maxillae appeared mildly hypoplastic yet symmetrically developed. Thoracic cage demonstrated a bell-shaped configuration with poorly developed upper part ( $\mathbf{F i g . 2 A}$ and $\mathbf{2 B}$ ). There were bilateral 11 pairs of ribs. Posterior ossification gaps were noted in the first right to sixth ribs and left second to fifth ribs. These posterior ossification gaps appeared as short segmental defects in the proximal parts of ribs. Proximal rib elements were hypoplastic, and showed an elongated neck. Rib margins at the gap showed conical tapering from both ends. Intervening ossified components had variable gap. No calcification or ossification was noted. Remaining ribs showed a spatulate (wider anteriorly) appearance (-Fig. 3). Involved chest segment was flail. On further 3D processing of the chest wall, a smooth pleural interface was seen at affected regions. There was a focal inner chest wall bulging with corresponding outpouching of pleura on right side ( - Fig. 2C). Upper lungs appeared small, underdeveloped, compared with rest of the lungs. Oropharyngeal airways and upper trachea were of normal capacity and configuration ( - Fig. 1C, 1D), whereas lower trachea was tapering distally, flattened in anteroposterior direction (-Fig. 2E, 2F). Spine evaluation (-Fig. 4) revealed the absence of ventral ossification center of atlas with nonfusion of posterior arches of rest of the cervical vertebrae. (-Fig. 1A, 1B) There was partial agenesis of $\mathrm{C} 4$ and $\mathrm{C} 5$ vertebral bodies. Butterfly vertebrae were noted at $\mathrm{T} 6$ and T7 levels (-Fig. 4C and $\mathbf{4 E}$ ); rest of the dorsal spine was normal. There was partial agenesis of L5 vertebral body. S1 vertebral body was ossified on right side, whereas rest of the sacral vertebral component was absent, likely indicating partial sacral agenesis. Posterior elements of all the vertebrae were nonfused. Both iliac bones were medially located due to sacral dysgenesis resulting in a pelvic deformity.

\section{Discussion}

Chest wall and airway involvement is the striking feature of CCMS, that often leads to precise diagnosis on radiography and alerts the neonatologist for possible difficult intubation and challenging respiratory support issues. In CCMS, the posterior aspects of the ribs are dysplastic because bone is replaced with cartilage or fibrous tissue that may eventually undergo calcification. ${ }^{5}$ Autopsy histological evaluation in another patient showed fibrovascular replacement of affected ribs. ${ }^{6}$ Extent of rib changes is variable from single focal posterior defect to extensive changes involving most of the 


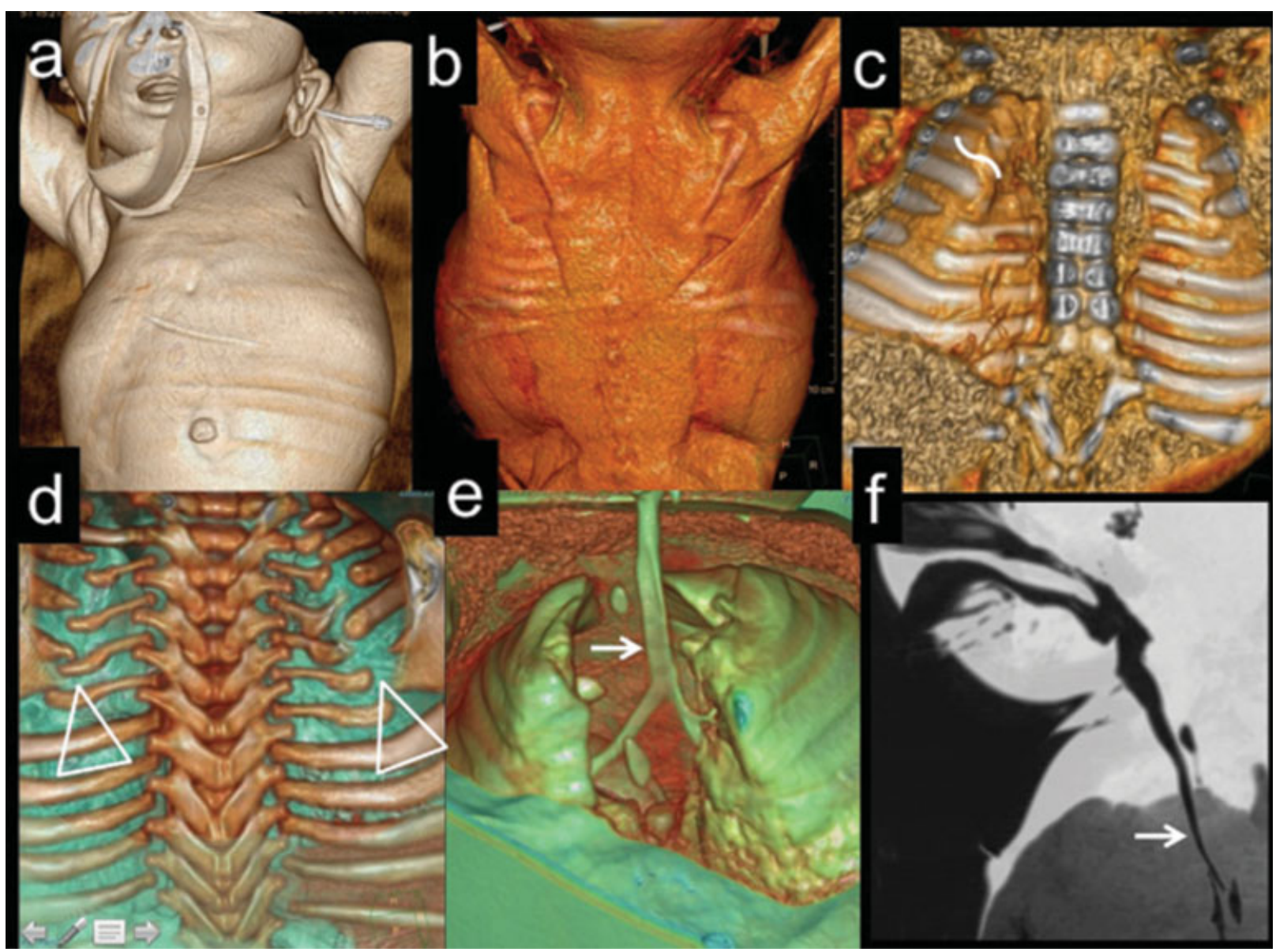

Fig. 2 Bell-shaped chest is demonstrated with small upper chest. Musculature grossly appears normal (A, B). Three-dimensional surface reconstruction ( $C$ and $D$ ) shows rib gap defects (triangle) and localized outpouching of chest wall (curved line). Three-dimensional and sagittal reconstruction of airways ( $\mathrm{E}$ and $\mathrm{F}$ ) show normal capacity upper airway and anteroposterior flattening of distal airway (arrows).

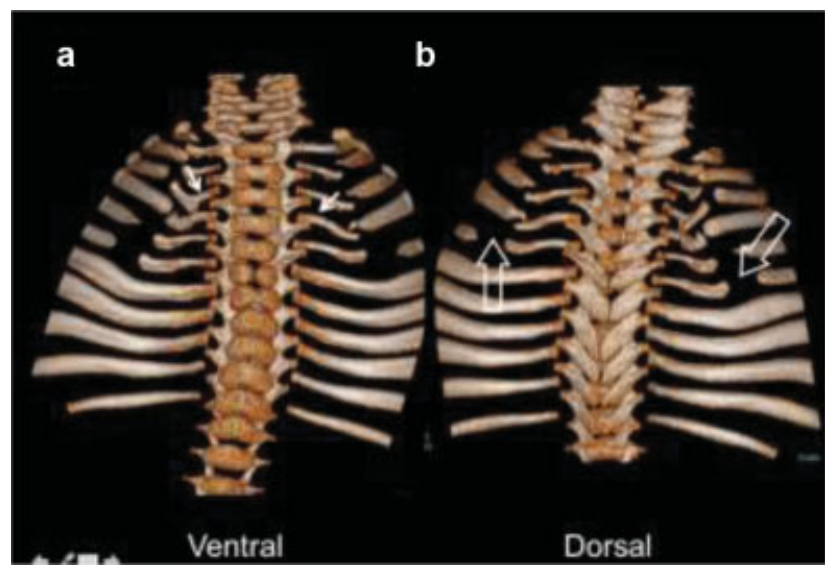

Fig. 3 Three-dimensional bone reconstructions from ventral $(A)$ and dorsal (B) perspective reveal multiple rib gap defects (open arrow). Proximal bone component is hypoplastic, deformed shows elongated neck (arrow). Lower ribs show wide anterior ends.

ribs, leading to flail chest. ${ }^{3,7}$ Forth to tenth ribs are usually involved. ${ }^{1}$ These defects often result in a bell-shaped thorax and may lead to underlying lung hypoplasia, compounding upper-airway obstruction. Abnormal costotransverse artic- ulations were shown on $\mathrm{CT}$ in a patient with extensive rib involvement. ${ }^{3}$ Postnatal growth retardation and microcephaly are common findings. ${ }^{3,8}$ Central nervous system changes of hypoxic ischemic nature can occur due to prolonged respiratory insufficiency. Other less common reported findings include hearing loss, tracheal, hyoid cartilage ossification abnormalities, ${ }^{3}$ choanal atresia, elbow and clavicular hypoplasia, scoliosis, pterygium colli, renal and central nervous system developmental disorders such as spina bifida. Renal anomalies like horseshoe kidney and hypospadias are reported. ${ }^{3}$ Cardiac defects are uncommon except for occasional septal defect. ${ }^{3}$ Early diagnosis of this rare syndrome can lead to early intervention and respiratory support that could avoid the cerebral effects of prolonged hypoxemia.

Our patient, presented with facial dysmorphic features, central hypotonia, developmental motor delay, and microcephaly. Although his preliminary brain examination was reported as normal, more sensitive examination like magnetic resonance imaging is needed to evaluate subtle changes. Constellations of facial and skeletal features were typical of CCMS. Our case appears to represent a less severe presentation because of relatively patent upper oropharyngeal airway. However, subtle changes of narrow distal airway 


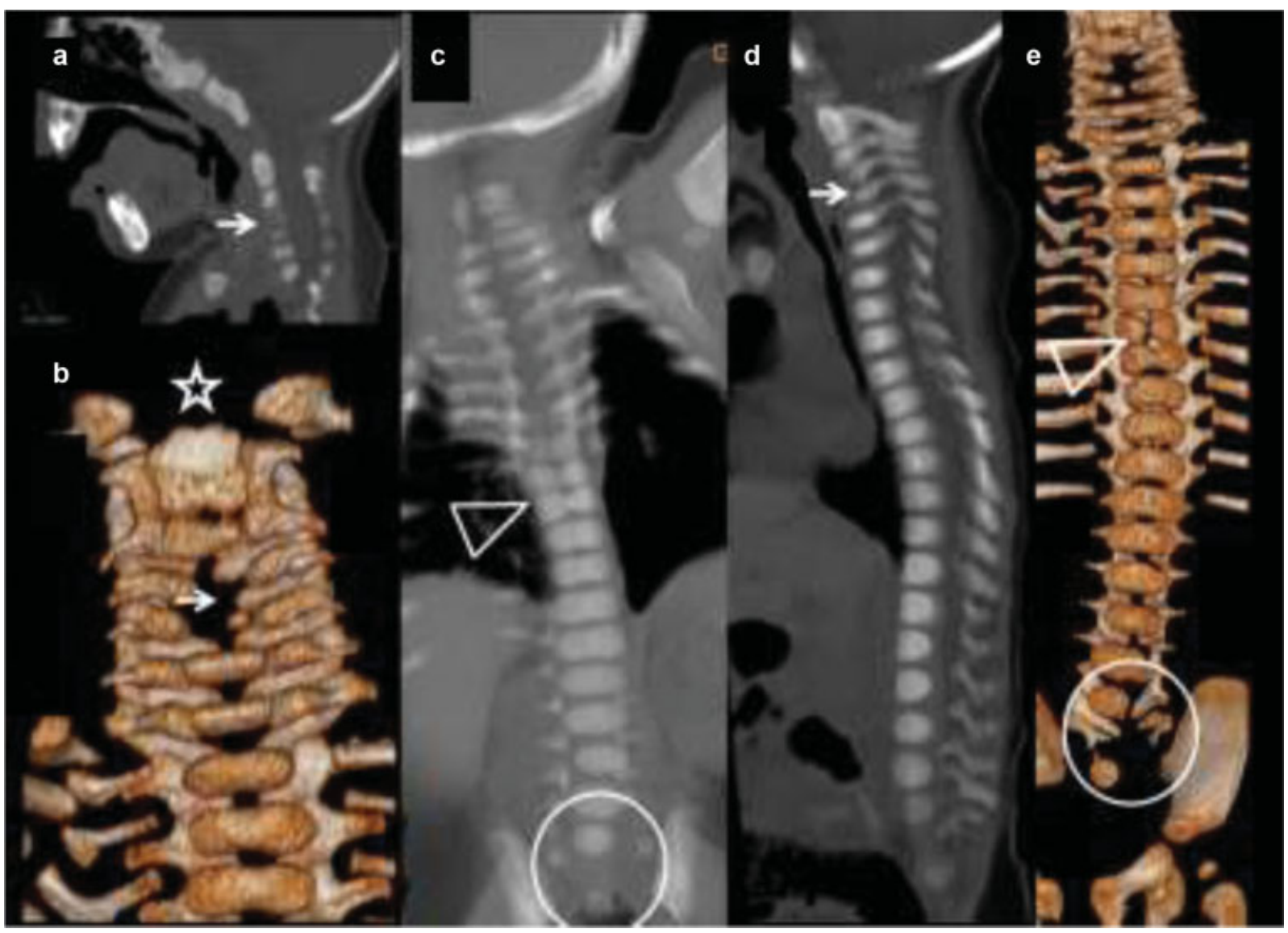

Fig. 4 Sagittal and three-dimensional (3D) views of cervical spine (A and B) show defective ossification of anterior and posterior arch of atlas (star). Also, there is hypoplastic bodies of C4, 5 (arrow). Coronal, sagittal, and 3D bone reconstructions of spine show butterfly vertebrae at T 6 and $T 7$ levels (triangle) (C and D). Also, there is agenesis of body of L5 and gross partial sacral agenesis (E) (circle).

and upper lung hypoplasia were observed on CT imaging. This information regarding airway is valuable as intubation in our patient is likely to be uneventful, although narrow distal airways and lung hypoplasia can impede the outcome of assisted ventilation. Additional information regarding the nature of rib defects as well as secondary changes in the chest wall was better delineated on CT. Larger gaps and greater out pouching of the chest wall were contributory factors for a flail chest. Spinal changes like sacral dysgenesis, vertebral body, and posterior element hypoplasia were dominant feature in our patient. However, there was no evidence of associated neural anomalies.

\section{Conflict of Interest}

None.

\section{References}

1 OMIM. Cerebrocostomandibular syndrome; CCMS, 117650(2017) Available at: https://www.omim.org/entry/117650. Accesed February 25, 2020
2 Ramaswamy P, Negus S, Homfray T, De Rooy L. Severe micrognathia with rib dysplasia: cerebro-costo-mandibular syndrome. Arch Dis Child Fetal Neonatal Ed 2016;101(01):F85. Doi: 10.1136/ archdischild-2014-307855

3 Tooley M, Lynch D, Bernier F, et al. Cerebro-costo-mandibular syndrome: clinical, radiological, and genetic findings. Am J Med Genet A 2016;170A(05):1115-1126

4 Smith DW, Theiler K, Schachenmann G. Rib-gap defect with micrognathia, malformed tracheal cartilages, and redundant skin: a new pattern of defective development. J Pediatr 1966;69(05):799-803

5 Williams HJ, Sane SM. Cerebro-costo-mandibular syndrome: long term follow-up of a patient and review of the literature. AJR Am J Roentgenol 1976;126(06):1223-1228

6 Oestreich AE, Stanek JW. Preautopsy imaging in cerebro-costomandibular syndrome. Pediatr Radiol 2010;40(Suppl 1):S50. Doi: 10.1007/s00247-010-1703-x

7 Miller KE, Allen RP, Davis WS. Rib gap defects with micrognathia. The cerebro-costo-mandibular syndrome-a Pierre Robin-like syndrome with rib dysplasia. Am J Roentgenol Radium Ther Nucl Med 1972;114(02):253-256

8 Wilcox BK, Tatum SA. Cerebro-costo-mandibular syndrome presenting as Pierre Robin sequence. Am J Otolaryngol 2004;25(04): 258-262 